\title{
O Princípio Cooperativo das Portas Abertas \\ e a participação de pessoas jurídicas em Cooperativas no Brasil
}

The open Membership and the legal persons as members of Cooperatives in Brazil

\author{
Ana Paula Andrade Ramos Rodrigues ${ }^{1}$ \\ Mario De Conto 2 \\ Faculdade de Tecnologia do Cooperativismo - ESCOOP (Brasil)
}

Sumário: I. Introdução. II. O princípio das «Portas Abertas» e a participação de pessoas jurídicas em Cooperativas III. A participação de pessoas jurídicas em Cooperativas no Direito Brasileiro. IV. O posicionamento dos órgãos de fiscalização tributária e do Conselho Administrativo de Recursos Fiscais acerca da matéria. V. Conclusões VI. Bibliografia

Summary: I. Introduction. II. The «Open Door» Principle and the participation of legal persons in Cooperatives. III. The participation of legal persons in cooperatives under the Brazilian Law. IV. The interpretation made by the Tax Regulation Organs and the Administrative Courts about the principle. V. Conclusions. VI. Bibliography.

Resumo: O presente texto objetiva analisar o conteúdo jurídico do Princípio da Adesão Livre e Voluntária —-também chamado de Princípio das Portas Abertas - notadamente no que concerne à participação de pessoas jurídicas em Cooperativas. Embora a participação de pessoas jurídicas em Cooperativas não seja afastada pelo Princípio das Portas Abertas, a matéria gera certa controvérsia, principalmente sob o argumento de que haveria incompatibilidade entre a ausência de objetivo de lucro da Cooperativa e a orientação ao lucro por parte das pessoas jurídicas associadas. No Direito Brasileiro a participação de pessoas jurídicas em Cooperativas é possível em caráter excepcional. No

1 Advogada. Gerente Jurídica da Organização das Cooperativas Brasileiras - OCB. Correo electrónico: ana.rodrigues@ocb.coop.br.

2 Doutor em Direito. Coordenador de Ensino, Pesquisa e Extensão da Faculdade de Tecnologia do Cooperativismo - ESCOOP. Professor de Direito Cooperativo em cursos de graduação e pós-graduação. Advogado. Gerente Jurídico do Sistema OCERGS-SESCOOP/RS. Correo electrónico: mario-deconto@ocergs.coop.br. 
país, os órgãos de fiscalização tributária tendem ao entendimento restritivo em relação à associação de pessoas jurídicas. Essa restrição encontra razão no conceito de "Ato Cooperativo», praticado entre Cooperativa e seus associados, ao qual a Constituição Federal atribui «tratamento tributário adequado». Não obstante às restrições dos órgãos fiscalizados, recentes decisões do CARF, Tribunal Administrativo entendem a possibilidade de associação de pessoas jurídicas e o reconhecimento do ato cooperativo, nas relações estabelecidas entre pessoas jurídicas associadas e Cooperativa.

Palavras chave: cooperativa, associação, pessoa jurídica

Abstract: The present text aims to analyze the legal content of the Principle of Voluntary and Open Membership —also called the Open Doors Principle - notably with regard to the participation of legal entities in Cooperatives. Although the participation of legal entities in Cooperatives is not excluded by the Open Doors Principle, the matter raises a certain controversy, mainly on the grounds that there would be an incompatibility between the lack of a profit objective of the Cooperative and the orientation to profit by legal persons associated. In Brazilian Law, the participation of legal entities in Cooperatives is possible on an exceptional basis. In the country, the tax inspection bodies tend to the restrictive understanding regarding the association of legal entities. This restriction is justified in the concept of "Ato Cooperativo", practiced between Cooperative and its associates, to which the Federal Constitution assigns "adequate tax treatment". Notwithstanding the restrictions of the audited bodies, recent decisions of CARF (an Administrative Court) consider the possibility of association of legal persons and the recognition of the "Ato Cooperativo», in the relations established between associated legal entities and the Cooperative.

Keywords: cooperative, membership, legal persons 


\section{Introdução}

Através dos Princípios Cooperativistas ${ }^{3}$, a Aliança Cooperativa Internacional declara os enunciados que devem nortear a atuação das Cooperativas nas diversas ordens legais. Através do Princípio da Adesão Livre e Voluntária - comumente chamado de Princípio das Portas Abertas - a ACl reconhece as cooperativas como organizações voluntárias, abertas a todas as pessoas hábeis a usar seus serviços e que desejem aceitar as reponsabilidades decorrentes da associação, sem discriminações sociais, raciais, políticas, religiosas ou de gênero.

A participação de pessoas jurídicas em Cooperativas, embora não afastada pelo Princípio das Portas Abertas, é matéria que gera certa con-

3 A Aliança Cooperativa Internacional reconhece os seguintes princípios cooperativistas: $1 .^{\circ}$-Adesão voluntária e livre; $2 .^{\circ}$-Gestão democrática; $3 .^{\circ}$-Participação econômica dos membros; $4 .^{\circ}$-Autonomia e independência; $5 .^{\circ}$-Educação, formação e informação; $6 .^{\circ}$ —Intercooperação; $7 .^{\circ}$ —Interesse pela comunidade. "CO-OPERATIVE PRINCIPLES: The co-operative principles are guidelines by which co-operatives put their values into practice. 1. Voluntary and Open Membership. Co-operatives are voluntary organisations, open to all persons able to use their services and willing to accept the responsibilities of membership, without gender, social, racial, political or religious discrimination. 2. Democratic Member Control. Co-operatives are democratic organisations controlled by their members, who actively participate in setting their policies and making decisions. Men and women serving as elected representatives are accountable to the membership. In primary co-operatives members have equal voting rights (one member, one vote) and co-operatives at other levels are also organised in a democratic manner. 3. Member Economic Participation. Members contribute equitably to, and democratically control, the capital of their co-operative. At least part of that capital is usually the common property of the co-operative. Members usually receive limited compensation, if any, on capital subscribed as a condition of membership. Members allocate surpluses for any or all of the following purposes: developing their co-operative, possibly by setting up reserves, part of which at least would be indivisible; benefiting members in proportion to their transactions with the co-operative; and supporting other activities approved by the membership. 4. Autonomy and Independence Co-operatives are autonomous, selfhelp organisations controlled by their members. If they enter into agreements with other organisations, including governments, or raise capital from external sources, they do so on terms that ensure democratic control by their members and maintain their co-operative autonomy. 5. Education, Training and Information. Co-operatives provide education and training for their members, elected representatives, managers, and employees so they can contribute effectively to the development of their co-operatives. They inform the general public - particularly young people and opinion leaders - about the nature and benefits of co-operation. 6. Co-operation among Co-operatives. Co-operatives serve their members most effectively and strengthen the co-operative movement by working together through local, national, regional and international structures.7. Concern for Community. Co-operatives work for the sustainable development of their communities through policies approved by their members». Aliança Cooperativa Internacional. "Statement on Cooperative Identity». Acesso em 24 de Maio de 2017. http://ica.coop/en/ whats-co-op/co-operative-identity-values-principles 
trovérsia no Direito comparado. O primeiro argumento contrário à participação de pessoas jurídicas em cooperativas está ligado à pretensa incompatibilidade entre a ausência de objetivo de lucro da Cooperativa e a orientação ao lucro por parte das pessoas jurídicas associadas.

No Direito Brasileiro, onde a participação de pessoas jurídicas em Cooperativas é possível em caráter excepcional, os órgãos de fiscalização tributária tendem ao entendimento restritivo em relação à associação de pessoas jurídicas. Essa restrição encontra razão no conceito de «Ato Cooperativo», instituto ao qual a Constituição Federal atribui tratamento tributário adequado e é restrito às operações realizadas entre Cooperativas e seus associados. Nesse sentido, considerar a possibilidade de associação de pessoas jurídicas em cooperativas acarreta reconhecer como «Ato Cooperativo» tais relações.

A controvérsia tem sido tratada em recentes decisões do Conselho Administrativo de Recursos Fiscais - CARF, que, ao contrário do entendimento dos órgãos de fiscalização tributária, tem se manifestado no sentido da possibilidade de associação de pessoas jurídicas e do reconhecimento do ato cooperativo, nas relações estabelecidas entre pessoas jurídicas associadas e Cooperativa.

\section{O princípio das «Portas Abertas» e a participação de pessoas jurídicas em Cooperativas}

A Aliança Cooperativa Internacional, através da Declaração sobre a Identidade Cooperativa, estabelece a definição de sociedade cooperativa, seus valores e princípios. No que concerne ao Princípio da "Adesão Livre e Voluntária», também chamado Princípio das portas abertas, a Declaração utiliza como critério para a associação a uma Cooperativa ser pessoa com aptidão para usar os serviços das Cooperativa, desejando aceitar as responsabilidades decorrentes da associação, sem discriminações sociais, raciais, políticas, religiosas ou de gênero. O princípio não faz distinção entre a associação de pessoas físicas e jurídicas.

A Declaração de Identidade Cooperativa adquiriu caráter normativo na ordem jurídica internacional a partir de sua recepção expressa na Recomendação 193/2002 da Organização Internacional do Trabalho, que trata da Promoção de Cooperativas ${ }^{4}$.

4 «3. The promotion and strengthening of the identity of cooperatives should be encouraged on the basis of: (...) (b) cooperative principles as developed by the international cooperative movement and as referred to in the Annex hereto. These principles 
No âmbito do Direito Comparado, as conclusões da Comissão encarregada do Projeto da Aliança Cooperativa Internacional denominado Princípios de Direito Cooperativo Europeu (PECOL), conclui que «associados são pessoas naturais ou jurídicas que participam das transações cooperativas como consumidores, fornecedores ou trabalhadores em empresa cooperativa» 5 .

Por outro lado, como adverte Hagen Henry, algumas ordens jurídicas restringem a participação de pessoas jurídicas em cooperativas singulares, baseadas em dois argumentos: (1) o de que a admissão de pessoas jurídicas que visam lucro em cooperativas é contraditória com a ideia de que a cooperativa não é uma entidade que visa o lucro; (2) o risco de que os sócios pessoas físicas sejam sobrepujados pelas pessoas jurídicas.

Em relação ao primeiro argumento, verifica-se a confusão da natureza jurídica da cooperativa e de seus membros. Com efeito, não obstante ao fato de que a Cooperativa não visa ao lucro, mas a prestação de serviços aos seus associados, é evidente que os sócios da Cooperativa buscam maximizar seus ganhos através do empreendimento coletivo.

Em relação ao segundo argumento, Hagen refere que (a) a admissão de pessoas jurídicas é voluntária e pode ser evitada dentro dos limi-

are: voluntary and open membership; democratic member control; member economic participation; autonomy and independence; education, training and information; cooperation among cooperatives; and concern for community». Organização Internacional do Trabalho. «Recomendação 193/2002». Acesso em 24 de Maio de 2017. http://www. ilo.org/dyn/normlex/en/f?p=NORMLEXPUB :12100:0::NO::P12100_ILO_CODE:R193.

5 (1) The members of a cooperative may be cooperator members or noncooperator members. (2) Cooperator members are natural or legal persons who engage in cooperative transactions as consumers, providers or workers of the cooperative enterprise. «Principles of European Cooperative Law (PECOL)» http://www.euricse.eu/wp-content/ uploads/2015/04/PECOL-May-2015.pdf

6 «However, many legislations exclude legal persons from membership in primary cooperatives. Generally, two types of arguments are put forward for this limitation: The first is to say that membership of capital centred legal entities in cooperatives is contradictory to the very idea of cooperatives being not-for-profit organizations. This argument confuses the nature of cooperatives and the motivation of the cooperative members. The second argument evokes the risk that physical person members will be overruled by legal person members. This risk is real, but it can be reduced as follows: 1 . Mixed membership is voluntary and can be avoided within the limits of the cooperative principles by physical person members who do not want to admit legal person members to the membership. 2. The voting power of legal person members, in those primary cooperatives which also have natural person members, can be limited so as not to allow these legal person members to outnumber the votes of the natural person members or to take decisions by themselves». HENRŸ, Hagen. "Guidelines for cooperative legislation» Geneva: ILO, 2012. 
tes dos princípios cooperativistas; (b) o poder de voto das pessoas jurídicas nas cooperativas singulares pode ser limitado, para não permitir às pessoas jurídicas superarem os votos das pessoas físicas.

\section{A participação de pessoas jurídicas em Cooperativas no Direito Brasileiro}

\section{a. Cooperativas em Geral}

No Direito Brasileiro, a Lei Geral de Cooperativas (Lei 5.764/71), estabelece a possibilidade de participação de pessoas jurídicas em Cooperativas, estabelecendo algumas restrições:

Art. $6{ }^{\circ}$ As sociedades cooperativas são consideradas:

I.- - singulares, as constituídas pelo número mínimo de 20 (vinte) pessoas físicas, sendo excepcionalmente permitida a admissão de pessoas jurídicas que tenham por objeto as mesmas ou correlatas atividades econômicas das pessoas físicas ou, ainda, aquelas sem fins lucrati$\operatorname{vos} ;(\ldots)$

Nesse sentido, a constituição de Cooperativas no ordenamento jurídico brasileiro é realizada, unicamente por pessoas físicas. Não há viabilidade jurídica, no ordenamento jurídico brasileiro, para a constituição de Cooperativas unicamente por pessoas jurídicas.

A lei faculta a admissão de pessoas jurídicas, em caráter excepcional, nos seguintes casos:

a) Pessoas jurídicas que tenham por objeto as mesmas ou correlatas atividades econômicas das pessoas físicas;

b) Pessoas jurídicas sem fins lucrativos.

A excepcionalidade prevista em lei apresenta um caráter dificultador, uma vez que a lei não apresenta parâmetros para sua definição. Além do caráter excepcional, limita a admissão de pessoas jurídicas em cooperativas singulares, o disposto no art. 29, apresenta outros requisitos:

Art. 29. O ingresso nas cooperativas é livre a todos que desejarem utilizar os serviços prestados pela sociedade, desde que adiram aos propósitos sociais e preencham as condições estabelecidas no estatuto, ressalvado o disposto no artigo $4 .^{\circ}$, item I, desta Lei.

(...) 
$\S 2 .^{\circ}$ Poderão ingressar nas cooperativas de pesca e nas constituídas por produtores rurais ou extrativistas, as pessoas jurídicas que pratiquem as mesmas atividades econômicas das pessoas físicas associadas.

$\S 3 .^{\circ}$ Nas cooperativas de eletrificação, irrigação e telecomunicações, poderão ingressar as pessoas jurídicas que se localizem na respectiva área de operações.

$\S 4 .^{\circ}$ Não poderão ingressar no quadro das cooperativas os agentes de comércio e empresários que operem no mesmo campo econômico da sociedade.

O art. 29, em seus $\S 2 .^{\circ}$ e $3 .^{\circ}$ apresentam exemplos de admissão de pessoas jurídicas em cooperativas, como em (1) cooperativas de pesca e nas constituídas por produtores rurais ou extrativistas e nas (2) Nas cooperativas de eletrificação, irrigação e telecomunicações.

A lei estabelece, ainda, em seu art. $29, \S 4 .^{\circ}$ a restrição ao ingresso dos agentes de comércio e empresários que operem no mesmo campo econômico da sociedade. Essa restrição relaciona-se à impossibilidade de que pessoas físicas ou jurídicas que exercem concorrência com a Cooperativa venham a se associar.

Dessa forma, segundo a lei geral de Cooperativas, a participação de pessoas jurídicas em cooperativas singulares está restrita, em caráter excepcional, às (1) pessoas jurídicas que tenham por objeto as mesmas ou correlatas atividades econômicas das pessoas físicas; às (2) pessoas jurídicas sem fins lucrativos, observando-se ainda que a pessoa jurídica não exerça atividades concorrenciais com a Cooperativa.

\section{b. Cooperativas de Crédito}

As Cooperativas de Crédito, embora submetidas à Lei Geral das Cooperativas, possuem dispositivos específicos no que concerne à matéria. Segundo o art. $4 .^{\circ}$ da Lei Complementar 130/2009, que disciplina as Cooperativas de Crédito, não há distinção entre a participação de pessoas físicas ou jurídicas, tampouco qualquer menção à excepcionalidade da participação de pessoas jurídicas.

Art. 4o O quadro social das cooperativas de crédito, composto de pessoas físicas e jurídicas, é definido pela assembléia geral, com previsão no estatuto social.

Parágrafo único. Não serão admitidas no quadro social da sociedade cooperativa de crédito pessoas jurídicas que possam exercer concorrência com a própria sociedade cooperativa, nem a União, os Es- 
tados, o Distrito Federal e os Municípios bem como suas respectivas autarquias, fundações e empresas estatais dependentes.

Relevante salientar que segundo dispõe o parágrafo único do art. $4 .^{\circ}$ da Lei Complementar 130/2009, há restrição quanto à associação de pessoas jurídicas que possam exercer concorrência com a Sociedade Cooperativa, bem como as pessoas jurídicas de direito público.

\section{O posicionamento dos órgãos de fiscalização tributária e do Conselho Administrativo de Recursos Fiscais acerca da matéria}

No Brasil, o questionamento referente à associação de pessoas jurídicas em Cooperativas tem sido tratado na seara dos órgãos de fiscalização tributária. A explicação para esse questionamento está diretamente ligado à extensão do conceito de "Ato Cooperativo».

Inicialmente, relevante ressaltar que a Constituição Federal de 1988 reconhece o papel do Cooperativismo, estabelecendo dispositivos para seu estímulo. Exemplos disso são dispositivos em que a Constituição Federal (1) veda a interferência estatal em Cooperativas, dispondo que «a criação de associações e, na forma da lei, a de cooperativas independem de autorização, sendo vedada a interferência estatal em seu funcionamento» (art. 5. ${ }^{\circ}$ XVIII); (2) reconhece a necessidade do «adequado tratamento tributário ao ato cooperativo praticado pelas sociedades cooperativas (art. 146, III, «C») e; (3) determina o papel do Estado, como agente normativo e regulador da atividade econômica, ao estabelecer que «a lei apoiará e estimulará o cooperativismo e outras formas de associativismo» (art. 174, § 2..$^{\circ}$ ).

O conceito de Ato Cooperativo é estabelecido pela Lei 5.764/71 e está vinculado às operações realizadas entre a Cooperativa e seus associados e vice-versa.

Art. 79. Denominam-se atos cooperativos os praticados entre as cooperativas e seus associados, entre estes e aquelas e pelas cooperativas entre si quando associados, para a consecução dos objetivos sociais.

Parágrafo único. O ato cooperativo não implica operação de mercado, nem contrato de compra e venda de produto ou mercadoria.

É nesse sentido que o entendimento dos órgãos de fiscalização tributária, no afã de afastar a configuração do "Ato Cooperativo», adotam postura restritiva quanto à associação de pessoas jurídicas em Co- 
operativas. A Receita Federal do Brasil, em posicionamento oficial7, apresenta o entendimento de que somente é «é possível o ingresso de pessoa jurídica nas sociedades cooperativas de pescas e nas cooperativas constituídas por produtores rurais ou extrativistas que pratiquem as mesmas atividades econômicas das pessoas físicas associadas». Trata-se de entendimento que restringe à associação em Cooperativas às duas hipóteses - ao nosso ver meramente exemplificativas - estabelecidas pela Lei 5764/71, em seus parágrafos $\S 2{ }^{\circ}$ e $\S 3 .^{\circ}$.

Esse entendimento $3 / 4$ ao nosso ver equivocado $3 / 4$ tem sido rechaçado pelo Conselho Administrativo de Recursos Fiscais em seus recen-

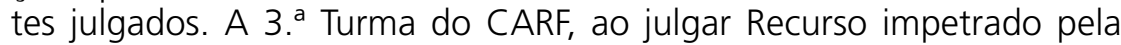
Fazenda Nacional em face de Cooperativa de Transporte, assim se manifestou:

ASSUNTO: CONTRIBUIÇÃO PARA O PIS/PASEP Período de apuração: 01/01/2008 a 31/12/2008 PIS. COFINS. ATO COOPERATIVO. PARTICIPAÇÃO DE PESSOA JURÍDICA COMO COOPERADO. NÃO INCIDÊNCIA. As pessoas jurdicas podem participar do quadro societário das cooperativas desde que respeitados os ditames do Código Civil e da Lei n.. ${ }^{\circ} 5.764 / 1971$.

Ato cooperado é aquele praticado entre as cooperativas e seus associados, entre estes e aquelas e pelas cooperativas entre si quando associados, para a consecução dos objetivos sociais (artigo 79 da Lei n.. 5.764/1971). A não incidencia de COFINS restringe-se a atos cooperados praticados entre a cooperativa e seus associados. Vistos, relatados e discutidos os presentes autos ${ }^{8}$.

Em seus argumentos, aduz ainda a Relatora:

No que diz respeito às pessoas jurídicas, tratam-se de pessoas jurídicas com fins lucrativos e que operam no ramo de transporte rodovi-

7 Quais as pessoas jurídicas que têm seu ingresso permitido nas sociedades cooperativas? Em situações específicas é possível o ingresso de pessoa jurídica nas sociedades cooperativas de pescas e nas cooperativas constituídas por produtores rurais ou extrativistas que pratiquem as mesmas atividades econômicas das pessoas físicas associadas. Exemplo: As microempresas rurais, os clubes de jovens rurais e os consórcios e condomínios agropecuários que praticarem agricultura, pecuária ou extração, desde que não operem no mesmo campo econômico das cooperativas. Ressalte-se que nas cooperativas de eletrificação, irrigação e telecomunicações, poderão ingressar as pessoas jurídicas que se localizem na respectiva área de operações. Receita Federal. https://idg.receita.fazenda.gov.br/orientacao/tributaria/declaracoes-e-demonstrativos/ecf-escrituracao-contabil-fiscal/perguntas-e-respostas-pessoa-juridica-2016-arquivos/capitulo-xvii-sociedadescooperativas-2016.pdf

8 Conselho Administrativo de Recursos Fiscais - CARF. Acórdao n. ${ }^{\circ} 9303-$ $004.358-3 .^{\text {a }}$ Turma 
ário de carga. Inclusive, e conforme a seguir demonstrará, parcela expressiva das exclusões da base de cálculo do PIS e da COFINS, e que foi levada a termo pelo sujeito passivo, se referem a repasses a asociados por conta de serviços de por eles prestados à cooperativa.

Para melhor compreensão das providências adotadas por esta autoridade + fiscal, convén esclarecer que a questão que deu gênese a presente autuação é o fato da COOPERCARGA excluir da base de cálculo do PIS e da COFINS os repasses efetuados aos asociados pessoas jurídicas. É de se concluir, outrossim, que cumprido está o requisito exigido no artigo $6 .^{\circ}$ da Lei $n .^{\circ} 5.764 / 71$, motivo pelo qual estamos diante da figura do ato cooperado. $\rangle^{9}$

Nesse sentido, a posição restritiva dos órgãos de fiscalização tributária que, ao restringir a participação de pessoas jurídicas em Cooperativas busca limitar a aplicação do conceito de Ato Cooperativo a tais relações tem sido rechaçada pela jurisprudência do Conselho Administrativo de Recursos Fiscais.

\section{Conclusões}

O Princípio das Portas Abertas não faz distinção no que concerne a participação de pessoas físicas ou jurídicas em Cooperativas. No Direito comparado, algumas ordens jurídicas estabelecem restrições a tal associação, notadamente em face da confusão entre ausência de finalidade lucrativa da cooperativa e a intenção de lucro das pessoas jurídicas associadas.

No Brasil, excetuando-se as Cooperativas de Crédito, nas demais cooperativas a lei estabelece que essa participação se dá em caráter excepcional. Essa disposição —a qual a lei não estabelece parâmetros objetivos - gera certa insegurança, na medida em que os órgãos de fiscalização tributária adotam posicionamento restritivo em relação a associação de pessoas jurídicas em Cooperativas, objetivando limitar o alcance do "Ato Cooperativo». As decisões do Conselho Administrativo de Recursos Fiscais tem sido favoráveis às Cooperativas, permitindo a associação de pessoas jurídicas e reconhecendo as operações realizadas como "Ato Cooperativo».

9 Conselho Administrativo de Recursos Fiscais - CARF. Acórdao n. ${ }^{\circ} 9303-$ $004.358-3$. $^{\text {a }}$ Turma 


\section{Bibliografia}

ALIANÇA COOPERATIVA INTERNACIONAL (ACI). «Guidance Notes to the Cooperative Principles». Acesso em 24 de Maio de 2017. http://ica.coop/en/ blueprint-themes/identity/guidancenotes

ALIANÇA COOPERATIVA INTERNACIONAL (ACI). «Statement on Cooperative Identity». Acesso em 24 de Maio de 2017. http://ica.coop/en/whats-co-op/ co-operative-identity-values-principles

ANDION, C., MORAES, R.L. y GONSALVES, A. 2017. «Organizaciones de la sociedad civil e innovación social ¿cómo y en qué medida influyen en el cambio social y político?», CIRIEC-España, Revista de Economía Pública, Social y Cooperativa, 90: 5-34.

BRASIL. 1971. «LEI 5.764, de 16 de dezembro de 1971». Acesso em 24 de Maio de 2017. http://www.planalto.gov.br/ccivil_03/leis/L5764.htm

ALIANÇA COOPERATIVA INTERNACIONAL (ACI). 1988. «Constituição da República Federativa do Brasil». Acesso em 24 de Maio de 2017. http:// www.planalto.gov.br/ccivil_03/Constituicao/Constituicao.htm

CONSELHO ADMINISTRATIVO DE RECURSOS FISCAIS - CARF. "Acórdao n. ${ }^{\circ} 9303004.358$ - 3. ${ }^{a}$ Turma». Acesso em 30 de Maio de 2017. https:// carf.fazenda.gov.br/sincon/public/pages/ConsultarJurisprudencia/consultarJurisprudenciaCarf.jsf

GÓMEZ CALVO, Verónica y GÓMEZ-ÁLVAREZ DÍAZ, Rosario. 2016. "La economía del bien común y la economía social y solidaria, ¿son teorías complementarias?. CIRIEC-España, Revista de Economía Pública, Social y Cooperativa, 87: 257-294.

HENRŸ, Hagen. 2012. «Guidelines for cooperative legislation» Geneva: ILO.

ORGANIZAÇÃO DAS NAÇÕES UNIDAS (ONU). «Declaração Universal dos Direitos Humanos» Acesso em 24 de Maio de 2017. http://www.ohchr.org/EN/ Professionallnterest/Pages/InternationalLaw.aspx

ORGANIZAÇÃO INTERNACIONAL DO TRABALHO (OIT). «Recomendação 193/2002». Acesso em 24 de Maio de 2017. http://www.ilo.org/dyn/normlex/en/f?p=NORMLEXPUB:12100:0::NO::P12100_ILO_CODE:R193

SARLET, Ingo Wolfgang. 2012. «Liberdade de associação cooperativa, direitos e garantias fundamentais e relações privadas na perspectiva do Supremo Tribunal Brasileiro». MEIRA, Deolinda Aparício Jurisprudência cooperativa comentada. Lisboa: INCM. 


\section{Derechos de autor}

El Boletín de la Asociación Internacional de Derecho Cooperativo es una revista de acceso abierto lo que significa que es de libre acceso en su integridad inmediatamente después de la publicación de cada número. Se permite su lectura, la búsqueda, descarga, distribución y reutilización legal en cualquier tipo de soporte sólo para fines no comerciales y según lo previsto por la ley; sin la previa autorización de la Editorial (Universidad de Deusto) o el autor, siempre que la obra original sea debidamente citada (número, año, páginas y DOI si procede) y cualquier cambio en el original esté claramente indicado.

\section{Copyright}

The International Association of Cooperative Law Journal is an Open Access journal which means that it is free for full and immediate access, reading, search, download, distribution, and lawful reuse in any medium only for non-commercial purposes, without prior permission from the Publisher or the author; provided the original work is properly cited and any changes to the original are clearly indicated. 